\title{
Effects of Geographic Variation in Vertical Mode Structure on the Sea Surface Topography, Energy, and Wind Forcing of Baroclinic Rossby Waves
}

\author{
DANiEl L. Codiga \\ Department of Marine Sciences, University of Connecticut, Groton, Connecticut \\ PETER CORNILLON \\ Graduate School of Oceanography, University of Rhode Island, Narragansett, Rhode Island
}

(Manuscript received 20 July 2001, in final form 10 December 2002)

\begin{abstract}
Interpretation of sea surface height anomaly (SSHA) and wind forcing of first baroclinic mode Rossby waves is considered using linear inviscid long-wave dynamics for both the standard and surface-intensified vertical mode in a continuously stratified rest-state ocean. The ratio between SSHA variance and vertically integrated energy of waves is proportional to 1) a dimensionless ratio characterizing the surface intensification of the pressure eigenfunction, 2) the squared internal gravity wave speed, and 3) the inverse of the water depth. Geographic variations in stratification and bathymetry can therefore cause geographically varying SSHA variance even for spatially uniform wave energy. The ratio between SSHA variance and wave energy across the North Atlantic shows important spatial variations based on eigensolutions for the standard vertical mode determined numerically using climatological hydrography. The surface-intensified mode result is similar, though the ratio is generally slightly larger and less sensitive to depth variations. Results are applied to the propagating annualfrequency portion of TOPEX altimeter SSHA in the North Atlantic. SSHA variance at $35^{\circ}$ in the western half of the basin increases by $\sim 63 \%$ over that in the east, but the associated change in inferred first-mode baroclinic Rossby wave energy is a substantially smaller increase of $\sim 26 \%(\sim 34 \%)$ for the standard (surface intensified) mode. This is mainly associated with increases to vertical mode surface intensification and squared internal gravity wave speed in the west due to stronger stratification above the pycnocline. The wind-forced wave equation for SSHA has a dimensionless coefficient of Ekman pumping that is proportional to the ratio between SSHA variance and wave energy, implying similar geographic variation in efficiency of wind excitation of Rossby wave SSHA.
\end{abstract}

\section{Introduction}

Rossby wave observations were limited [White 1977; Price and Magaard 1980; Halliwell et al. 1991; and others; Dewar (1998) includes a review] until sea surface height anomaly (SSHA) measurements by the TOPEX altimeter obtained improved spatial and temporal coverage to enable a more complete description of the signal (e.g., Chelton and Schlax 1996, hereinafter CS; Polito and Cornillon 1997, hereinafter PC). In many of the world's oceans a component of SSHA with dominantly westward propagation of a somewhat regular train of highs and lows (e.g., Fig. 2 of CS; Fig. 1 of PC) can be identified traveling across the basin at a nearly annual frequency. Because the observed speeds (CS; PC) are similar to that of the first baroclinic mode calculated using climatological hydrography (e.g., Emery et al.

Corresponding author address: Dr. Daniel L. Codiga, Department of Marine Sciences, University of Connecticut, 1080 Shennecossett Rd., Groton, CT 06340-6097.

E-mail: d.codiga@uconn.edu
1984; Chelton et al. 1998) and differ substantially from those of the barotropic and higher baroclinic modes, the signal is interpreted as the surface signature of first baroclinic mode Rossby waves. While the total SSHA field includes wave and nonwave contributions from each of the vertical modes (barotropic and all baroclinic), comparisons to moored current meters (e.g., Wunsch 1997) suggest that the barotropic and first baroclinic modes are the most important. The focus in this study is the component of SSHA due to first baroclinic mode, long Rossby waves at off-equatorial latitudes. This can be separated from standing and eastward-propagating components, which are generally less prominent, using sophisticated analysis approaches (e.g., PC; Vivier et al. 1999; Polito et al. 2000); here as in CS the signal is isolated, albeit imperfectly, by limiting attention to the annual frequency after application of a zonal high-pass filter to remove the nonpropagating steric component.

A striking attribute of this portion of the SSHA signal is geographic structure in its variance. Rich detailed patterns are seen in the North Atlantic (e.g., PC), Pacific 
(e.g., Vivier et al. 1999), and Indian (e.g., Wang et al. 2001) Oceans. Vivier et al. and Wang et al. interpret this as changes in wave energy. Although it may, in fact, result from variations in wave energy, it could also result from geographic variations in the vertical structure of the first baroclinic mode due to changes in stratification profile and water depth. A primary aim of this study is to investigate the importance of the latter effects. Wave propagation through a varying medium is well known to involve changes in wave amplitude while the integrated or total energy may be preserved (e.g., Lighthill 1978). This applies to baroclinic Rossby waves moving through geographically varying stratification profiles and water depths. Our main focus is the relation between SSHA variance, the wave attribute directly measured by altimeter, and vertically integrated wave energy; for example, we provide an estimate of the geographic structure of SSHA variance in the North Atlantic for waves of spatially uniform energy.

In addition to geographic structure in variance of propagating SSHA, observed speeds are systematically somewhat higher (CS; PC) than for the standard theory, and these attributes have together helped prompt a number of theoretical and modeling studies that modify the standard theory with additional processes. We use "standard theory" to refer to inviscid, linear, hydrostatic, Boussinesq, nearly geostrophic Rossby waves in a beta-plane, resting background state, flat-bottom ocean of geographically uniform stratification profile, with rigid-lid surface and kinematic seafloor boundary conditions; we refer to the vertical structure of such wave solutions as the "standard mode." Modifications to the standard theory include mean flow influences (e.g., Killworth et al. 1997; Dewar 1998), the impact of friction (e.g., Qiu et al. 1997), interaction between waves and topography (e.g., Killworth and Blundell 1999), and the effects of lateral and bottom boundaries and associated mode coupling (e.g., Pedlosky 2000; LaCasce 2000; Tailleux and McWilliams 2000, 2001). Because the present aim is to assess the importance of geographic variations in the first-mode eigensolutions, the initial approach treats climatological hydrographic data in terms of the unmodified standard theory. The intent is not to argue that modifications to the theory are unnecessary, but rather to use the standard theory as an initial evaluation of the effect. Then, to assess the robustness of the results to the use of the standard mode, we use the same hydrography to treat the surface-intensified vertical mode (Rhines 1970; Samelson 1992; Tailleux and McWilliams 2001; and others) for comparison. The surface-intensified mode is related to the standard mode by a changed bottom boundary condition that becomes appropriate, for example, to incorporate effects of sloping topography; one reason why it is of interest is that its speed exceeds that of the standard mode by an amount (Tailleux and McWilliams 2001) similar to observed SSHA propagation speeds.

The forced wave equation for SSHA is considered here as motivated by a number of studies (e.g., Sturges et al. 1998; Vivier et al. 1999; Wang et al. 2001; Birol and Morrow 2001) that demonstrate important control over Rossby wave SSHA by direct Ekman pumping (wind stress curl fluctuations in basin interiors away from boundaries). A unitless coefficient of Ekman pumping is identified and shown to have substantial geographic variations.

\section{Relating SSHA, wave energy, and wind forcing}

The standard theory (e.g., Rossby 1939; Gill 1982; Pedlosky 1987) for wind-forced baroclinic Rossby waves is used in this section to present 1) the ratio $\gamma$ between SSHA variance and vertically integrated wave energy and 2) a unitless coefficient $\alpha$ of the Ekman pumping forcing term in the wave equation for SSHA. Using standard notation, the governing momentum, density conservation, and continuity equations are

$$
\begin{aligned}
-f v & =-p_{x}+\left(1 / \rho_{o}\right) d \tau^{x} / d z \\
f u & =-p_{y}+\left(1 / \rho_{o}\right) d \tau^{y} / d z \\
0 & =-p_{z}+b \\
b_{t}+N^{2} w & =0 \\
u_{x}+v_{y}+w_{z} & =0 .
\end{aligned}
$$

Horizontal stresses are $\left[\tau^{x}(z), \tau^{y}(z)\right]$ and surface wind stress is $\tau^{\mathrm{o}}=\left(\tau^{\mathrm{ox}}, \tau^{\mathrm{oy}}\right)=\left[\tau^{x}(z=0), \tau^{y}(z=0)\right]$.

\section{a. Unforced system}

An infinite series of baroclinic normal modes (e.g., Gill 1982; Wunsch and Stammer 1997; Frankignoul et al. 1997) is considered (summations are over all $i$; real part understood):

$$
\begin{aligned}
p(x, y, z, t) & =\sum P_{i}(x, y, t) F_{i}(z) \\
w(x, y, z, t) & =\sum P_{i}(x, y, t) G_{i}(z) .
\end{aligned}
$$

Though a specific choice of normalization for $F$ and $G$ is used throughout this paper, the resulting expression for the ratio between SSHA variance and wave energy is provided in normalization-independent form below (11). The $i$ th baroclinic mode reduced pressure has temporal-horizontal function $P_{i}$, of dimensions length ${ }^{2} /$ time $^{2}$, and dimensionless vertical eigenfunction $F_{i}(z)$. The vertical velocity has vertical eigenfunction $G_{i}(z)$, of dimensions time/length. Modes are orthogonal to each other in a vertically averaged sense,

$$
(1 / H) \int_{-H}^{0}\left|F_{i}(z) F_{j}(z)\right| d z=\delta_{i j},
$$

for water depth $H$ and Kronecker delta $\delta_{i j}$. Our interest lies with the first baroclinic mode, which contributes most strongly to the sea surface height (e.g., Wunsch and Gill 1976; Wunsch 1997), so $i$ subscripts are 
dropped henceforth except where noted, and equations are understood to be for the first mode. The two eigenfunctions are related by

$$
F=\left(c^{2} / i \sigma\right) d G / d z
$$

where $c$ is the internal gravity wave speed and $\sigma[2 \pi$ $(\mathrm{yr})^{-1}$ throughout this study] is the wave frequency, for wave solutions $P(x, y, t) \propto \exp [i(k x-\sigma t)]$ with wavenumber $k$ in the $x$ direction, and our normalization includes the relation

$$
\int_{-H}^{0}|F(z)|^{2} d z=H=\left(c^{2} / \sigma^{2}\right) \int_{-H}^{0} N(z)^{2} G(z)^{2} d z .
$$

Separation of variables yields the separation constant $-1 / c^{2}$ and horizontal structure equation

$$
P_{t}+c_{R} P_{x}=0
$$

with westward phase speed for long Rossby waves $c_{R}$ $=-\beta c^{2} / f^{2}$. The vertical structure equation and boundary conditions are

$$
\begin{aligned}
G_{z z}+\left(N^{2} / c^{2}\right) G & =0 \\
G & =0 \text { at } z=0 \text { and } z=-H,
\end{aligned}
$$

the latter corresponding physically to zero vertical velocity conditions at the surface, where the rigid-lid assumption applies, and at the seafloor. In practice, for arbitrary stratification profile $N^{2}(z)$, the separation constant and eigenfunctions of system (6) are determined numerically.

We seek to relate the signature of the waves observed by altimeter, a component $\eta$ of SSHA, to the vertically integrated wave energy. Under the rigid-lid assumption the SSHA is a passive expression of the wave,

$$
\eta=p(z=0) / g=F(0) P / g,
$$

and for harmonic time dependence the time-average $\left(\langle\rangle^{t}\right)$ squared SSHA is therefore

$$
\left\langle\eta^{2}\right\rangle^{t}=\frac{1}{2} \frac{|F(0)|^{2}|P|^{2}}{g^{2}} .
$$

For the long waves of interest, potential energy dominates and takes the per-unit-mass form (1/2) $\int_{-H}^{0} b^{2} / N^{2}$ $d z$ (e.g., Pedlosky 1987). With use of (1c) and (4), the time-average energy per unit surface area, denoted $E$ (dimensions length ${ }^{3} /$ time $^{2}$ ), is then

$$
E=\left\langle\frac{1}{2} \int_{-H}^{0} \frac{b^{2}}{N^{2}} d z\right\rangle^{t}=\frac{1}{4} \frac{|P|^{2} H}{c^{2}} .
$$

It follows from (8) and (9) that $\left\langle\eta^{2}\right\rangle^{t}=\gamma E$, where $\gamma$ $\equiv\left(2 / g^{2}\right)|F(0)|^{2} c^{2} / H$. Using the dimensionless ratio

$$
I=|F(0)|^{2} /\left[(1 / H) \int_{-H}^{0}|F(z)|^{2} d z\right],
$$

a form for $\gamma$ that is independent of the choice of normalization results:

$$
\gamma=\left(2 / g^{2}\right) I c^{2} / H
$$

Equation (11) makes clear the dependence of SSHA variance, for a wave energy level $E$, on three parameters: it is (i) proportional to $I$, the normalized squared surface value of the pressure eigenfunction, (ii) proportional to the square $c^{2}$ of the internal gravity wave phase speed, and (iii) inversely proportional to the water depth $H$.

In the limit of an $N(z)$ profile that varies slowly with depth in a Wentzel-Kramers-Brillouin (WKB) sense, it can be shown (following, e.g., Chelton et al. 1998; Tailleux and McWilliams 2001) that

$$
\gamma_{\mathrm{WKB}}=4 N(0)\langle N\rangle^{z} H /\left(\pi^{2} g^{2}\right),
$$

where \langle\rangle$^{z}$ is the vertical mean. The expression makes clear that higher $\gamma$ will occur for stratification intensified at the surface, higher vertical-mean stratification, and deeper water. The difficulty inherent in selecting $N(0)$ appropriate to observed profiles, in which the WKB limit is violated most strongly near the surface due to rapid vertical variations associated with very low and high values within and below the mixed layer, respectively, makes evaluating (12) with measurements impractical. Therefore, the remaining analysis uses values of $\gamma$ (11) based on $I$ and $c$ values from numerically determined eigensolutions using climatological $N(z)$ profiles.

\section{b. Wind forcing}

Effects of zero-mean fluctuating wind stress curl in generating waves are considered. The forcing terms (1) are expanded (e.g., Gill 1982) in the baroclinic modes, for example, in the $x$-momentum equation as

$$
\frac{1}{\rho_{o}} \frac{d \tau^{x}}{d z}=\sum T_{i}^{x}(x, y, t) F_{i}(z)
$$

where

$$
T_{i}^{x}=\left[1 /\left(\rho_{o} H\right)\right] \int_{-H}^{0} F_{i}(z) d \tau^{x} / d z d z .
$$

The stress falls from its surface value to zero in the upper few hundred meters of the water column and over these depths $F(z)$ for the first mode is nearly uniform and close to its surface value, so $T_{i}^{x}=\tau^{x o} F_{i}(0) /\left(\rho_{\mathrm{o}} H\right)$ is a good approximation. The resulting forced version of the wave equation for $P(5)$ is

$$
P_{t}+c_{R} P_{x}=\left[c^{2}|F(0)| / H\right] w^{E},
$$

where $w^{E}=\left(1 / \rho_{\mathrm{o}}\right) \boldsymbol{\nabla} \times\left(\tau^{\circ} / f\right)$ is the Ekman pumping vertical velocity. We emphasize that (13) governs pressure eigenfunction $P$, not SSHA. By (7), the forced wave equation for SSHA is

$$
\eta_{t}+c_{R} \eta_{x}=\alpha w^{E}
$$

where the unitless coefficient of the forcing term, or Ekman pumping efficiency, is 

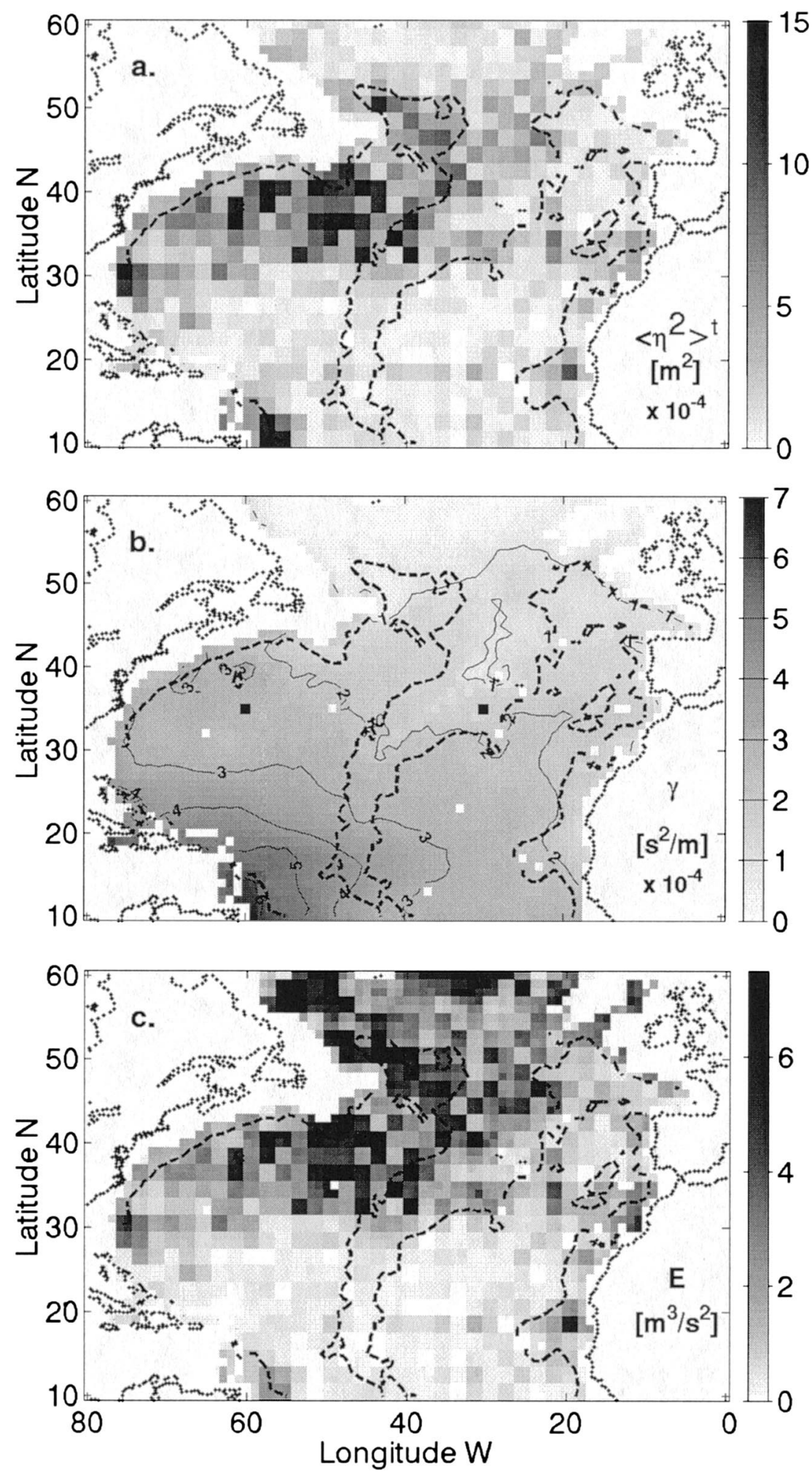

FIG. 1. (a) Variance $\left\langle\eta^{2}\right\rangle^{t}$ of propagating annual SSHA signature of first baroclinic mode Rossby waves in the North Atlantic based on TOPEX/Poseidon altimeter data between Oct 1992 and Jun 1997. In this figure and those to follow, heavy dashed contours mark the 4000$\mathrm{m}$ isobath (Fig. 3c). At $35^{\circ} \mathrm{N}$ values in the western half of the basin are a factor of $\sim 1.63$ larger than in the eastern half. (b) Ratio $\gamma$ of SSHA variance to vertically integrated energy $E$ of the first baroclinic mode, calculated from standard rest-state vertical mode eigensolutions 


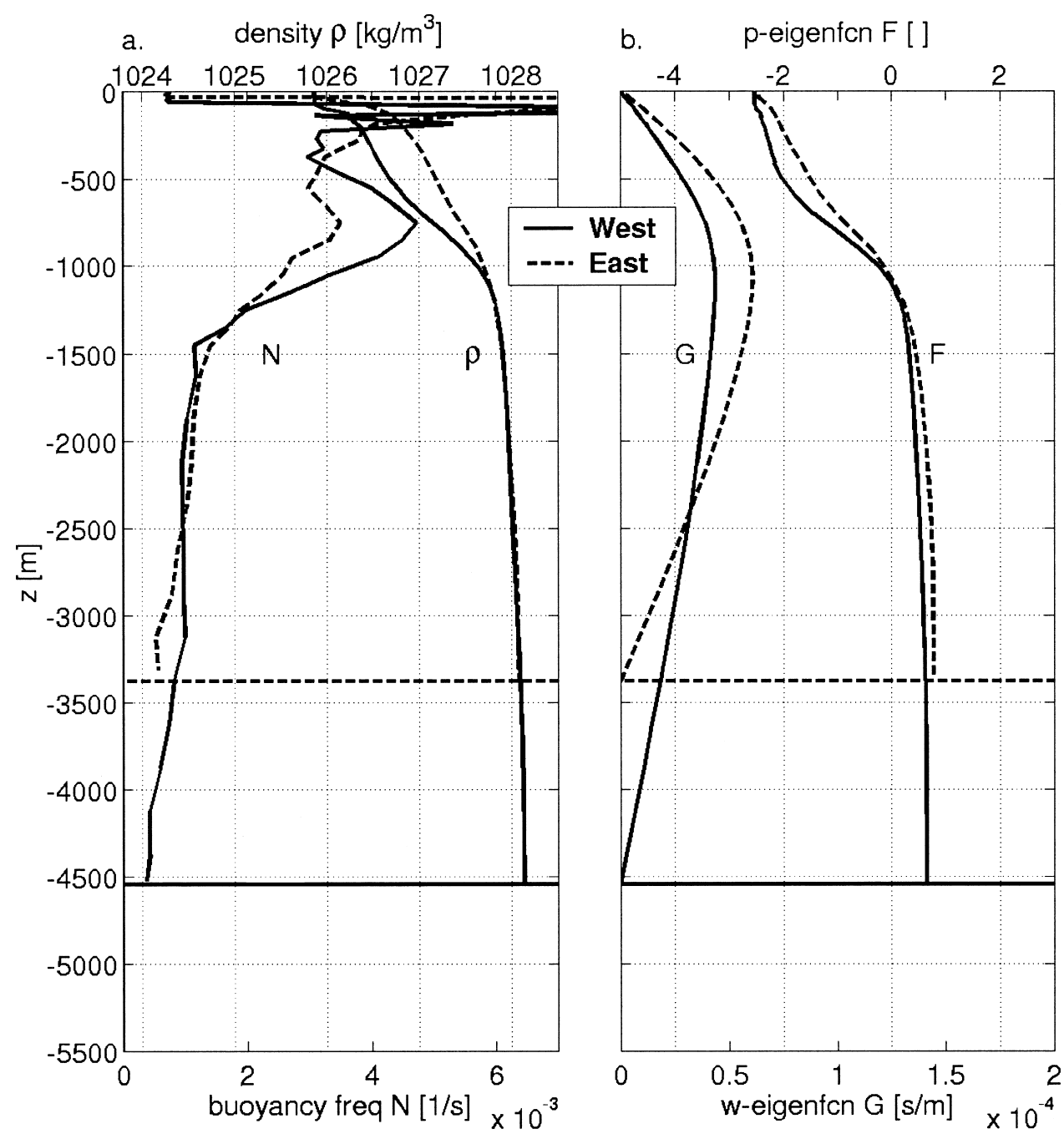

FIG. 2. Comparison between $35^{\circ} \mathrm{N}$ vertical mode structure at two representative locations marked by black squares in Fig. $1 \mathrm{~b}$, one to the west (long $60^{\circ} \mathrm{W}$, solid lines) and one to the east ( $30^{\circ} \mathrm{W}$, dashed lines) of the Mid-Atlantic Ridge. Horizontal lines mark seafloor depth. (left) Density $\rho$ (lower axis) and buoyancy frequency $N$ (upper axis) profiles; (right) first baroclinic mode vertical velocity eigenfunction $G$ (lower axis) and reduced pressure eigenfunction $F$ (upper axis) profiles.

$$
\alpha=(g / 2) \gamma \text {. }
$$

The physical meaning of $\alpha$ is that SSHA of the first baroclinic mode will be preferentially excited, by a given Ekman pumping, for conditions that result in large $\alpha$ values.

FIG. 1. (Continued) using climatological hydrography. Values span roughly an order of magnitude with geographic structure due to variations in the stratification profile and water depth. Black squares mark locations of profiles in Fig. 2. (c) First baroclinic mode Rossby wave energy $E$ inferred as the ratio $\left\langle\eta^{2}\right\rangle^{t} / \gamma$. At $35^{\circ} \mathrm{N}$ values in the western half of the basin are a factor of $\sim 1.26$ higher than in the eastern half, a westward increase $\sim 41 \%$ as high as that seen in $\left\langle\eta^{2}\right\rangle^{t}$.

\section{Results for standard vertical mode}

This section includes evaluation and examination of the spatial structure of the ratio $\gamma$ between SSHA variance and wave energy, and hence of the Ekman pumping efficiency $\alpha$. While the above derivations are for a standard flat-bottom ocean with arbitrary but horizontally uniform density profile, we now treat the more general situation where the bottom depth and the density profile vary geographically. The above analysis is presumed valid locally, which is strictly applicable only for geographic variations in stratification, depth, and Ekman pumping that are weak in a WKB sense, and this restriction is clearly not obeyed by the observed fields. Our intent is not to argue that this assumption applies to measured fields but rather to use it, because 
inclusion of non-WKB geographic variations is substantially more complicated, as a first step to explore the range of variation of the parameters of interest. The main points regarding interpretation of SSHA in relation to wave energy and wind forcing apply to all oceans; for demonstration the focus is annual frequency fluctuations in the North Atlantic between $10^{\circ}$ and $60^{\circ} \mathrm{N}$ excluding locations off the Mid-Atlantic Ridge less than $1500 \mathrm{~m}$ deep.

\section{a. Data and methods}

The analysis steps for SSHA from altimetry follow closely those of CS and PC. Merged geophysical data records from TOPEX/Poseidon for residual sea surface anomaly are used with standard choices for atmospheric corrections and removal of tides, inverse barometer effect, and mean sea surface (e.g., Benada 1997). The time period extends from October 1992 to June 1997. Data reduction includes screening of outliers, application of an along-orbit low-pass Butterworth filter to suppress scales smaller than $90 \mathrm{~km}$, and removal of the time mean. Values are interpolated with inverse-radius weighting to a $1^{\circ} \times 1^{\circ}$ grid. The propagating annual component is isolated by suppression of the nearly annual, large zonal scale portion associated with the steric component of the annual heating/cooling cycle (Stammer 1997) using a zonal high-pass Butterworth filter with $45^{\circ}$ cutoff, leaving a dominantly westward propagating signal. A least squares fit is made to a single annual harmonic at each grid point. Differences between results of the harmonic fit and a Butterworth temporal bandpass filter that suppresses energy outside the 280450 -day range of periods are typically several percent in SSHA. A $2^{\circ} \times 2^{\circ}$ average is applied to $\left\langle\eta^{2}\right\rangle^{t}$ (Fig. 1a) from the harmonic fit.

The hydrographic climatology used is the World Ocean Circulation Experiment (WOCE) Hydrographic Program Special Analysis Centre climatological hydrographic database (Gouretski and Jahncke 1998). This database builds on the World Ocean Atlas (Levitus et al. 1994) climatology of the National Oceanographic Data Center with respect to objective interpolation on neutral surfaces, vertical resolution and treatment of near-bottom values, inclusion of modern WOCE data, and certain aspects of quality control. The numerical eigensolution method is carried out at each point on the $1^{\circ} \times 1^{\circ}$ grid following the centered-first difference, neutral density gradient method described by Chelton et al. (1998).

\section{b. SSHA and inferred wave energy}

Geographic changes in the variance of the propagating annual component of SSHA are substantial across the North Atlantic (Fig. 1a). Maximum values are found in the western portion of the basin, particularly at latitudes from $30^{\circ}$ to $45^{\circ} \mathrm{N}$.
Based on numerical eigensolutions using climatological hydrography, $\gamma$ varies by roughly an order of magnitude across the North Atlantic (Fig. 1b). Maximum values are reached in the south and west and minima are found at high latitudes. At midlatitudes, the dominant structure is an increase to the west. The implication is that, if the energy in first-mode baroclinic Rossby waves was completely uniform geographically, the associated SSHA variance would nonetheless increase from east to west. As a result, interpreting SSHA variance as a direct indicator of wave energy [e.g., as for the Pacific by Vivier et al. (1999)], in effect presuming the two are related by a geographically uniform proportionality constant, would lead to the conclusion that the wave energy increases westward far more so than is the case.

On the other hand, using measured SSHA variance with $\gamma$ calculated from hydrographic climatology, the energy in the first baroclinic mode can be inferred. An example of the features revealed by the resulting inferred first-mode Rossby wave energy in the North Atlantic (Fig. 1c) is that at $35^{\circ} \mathrm{N}$ wave energy in the western half of the basin is a factor of 1.26 higher than in the eastern half. This westward increase is $41 \%$ as high as that seen in SSHA variance, which increases from east to west by a factor of 1.63 (Fig. 1a).

\section{c. Representative vertical modes east and west of the Mid-Atlantic Ridge}

To help to understand the origin of spatial variations in the ratio between SSHA variance and wave energy, we present eigensolutions for two North Atlantic locations at latitude $35^{\circ} \mathrm{N}$ (Fig. 2). One site is west of the Mid-Atlantic Ridge $\left(60^{\circ} \mathrm{W}, \sim 4550 \mathrm{~m}\right.$ deep $)$ and the other east of it $\left(30^{\circ} \mathrm{W}, \sim 3380 \mathrm{~m}\right.$ deep $)$. While densities deeper than the base of the pycnocline are very similar in the east and west, at shallower depths densities are lower in the west. This gives rise to higher buoyancy frequency through much of the upper water column in the west. The first-mode internal gravity wave speed $c$ is larger at the west site $\left(2.85 \mathrm{~m} \mathrm{~s}^{-1}\right)$ than at the east site $\left(2.16 \mathrm{~m} \mathrm{~s}^{-1}\right)$. This may be understood in terms of the fundamental dependence of $c$ on the vertical integral of buoyancy frequency and on depth (e.g., Chelton et al. 1998), given the stronger stratification and deeper water at the west site. The westward increase in $c$ is most responsible for $\gamma_{\text {west }} / \gamma_{\text {east }}=1.39$. Note also that near the surface, in the west, $|d G / d z|$ is smaller, but $|F|$ is larger as a result (3) of the larger $c^{2}$; deeper than about $1000 \mathrm{~m},|d G / d z|$ remains smaller in the west, but here $|F|$ is also smaller in the west despite the larger $c^{2}$.

\section{d. Factors contributing to the structure of $\gamma$}

The main aspects of the geographic structure of the ratio $\gamma$ (Fig. 1b) of SSHA variance to wave energy can 

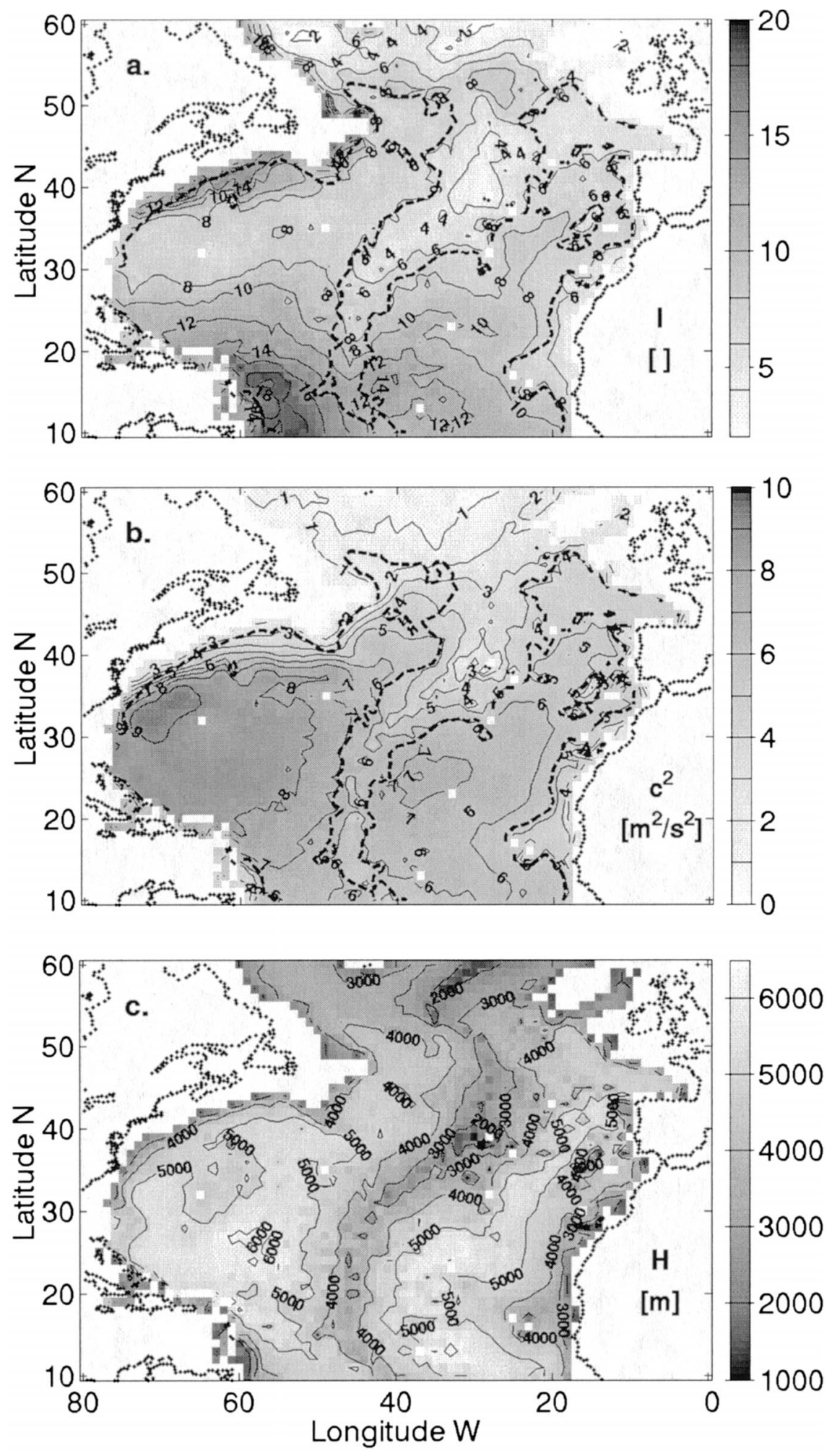

FIG. 3. Factors that contribute to $\gamma$ for the first baroclinic mode (shown in Fig. 1b): $\gamma$ is proportional to (a) $\times$ (b)/(c). (a) Normalized squared surface value of the pressure eigenfunction $I$, calculated from hydrographic climatology. (b) Squared internal gravity wave speed $c^{2}$. (c) Bathymetric depth $H$. 
be described as a combination of two broad patterns: a general increase from the northeast to the southwest and low values locally over regions of relatively shallow water. To understand the contributing effects, examine the factors that appear in the expression for $\gamma$ in (11): $I, c^{2}$, and $H$ (Fig. 3). The normalized squared surface pressure eigenfunction I (Fig. 3a) is controlled by both the water depth and the stratification profile through their impacts on the eigensolution. It shows underlying influences of Mid-Atlantic Ridge bathymetry as relatively low values over shallow water, particularly at latitudes $30^{\circ}-50^{\circ} \mathrm{N}$. In contrast, large $I$ values in the southwest, for example, are dominantly due to increased stratification shallower than the main pycnocline there. Both of these attributes in the structure of $I$ can be seen clearly in $\gamma$. The patterns in $c^{2}$ and $H$ (Figs. 3b,c) are similar to each other; they include small values paralleling the Mid-Atlantic Ridge, with relatively larger $c^{2}$ values in the west. The dependence of $\gamma$ is on the ratio $c^{2} / H$ (not shown), which appears to have less impact than $I$ except for the increased western values. In summary, the ratio of SSHA variance to wave energy (Fig. 1b) shows a broad increase towards the southwest and slightly reduced values along the Mid-Atlantic Ridge, both of which are mainly due to similar patterns in the normalized squared surface pressure eigenfunction.

\section{Results for surface-intensified mode}

A test of the robustness of the above results to the use of the standard theory for vertical modes is appropriate. For example, Killworth and Blundell (1999) developed modifications to vertical-mode eigensolutions due to topography when the stratification profile is horizontally uniform, and included a relation between energy and SSHA equivalent to (11) except for the use of their modified eigensolutions. Extensions to the standard theory can be incorporated in $\gamma$ in some cases by replacement of $I$ and $c$ with values using the modified mode structure. In this section we carry this out for the "surface intensified" vertical mode. The surface-intensified mode, a modified form of the standard mode that applies in the presence of steep bottom topography, has been explored by Rhines (1970), Samelson (1992), and others. Tailleux and McWilliams (2001) identify a "bottom-pressure decoupled" mode as the surface-intensified mode, emphasizing that its higher speed may account SSHA observations.

The surface-intensified ("si") mode obeys the equations of section 2 with the exception that the bottom boundary condition (6b) at $z=-H$, namely, $G=0$, is replaced by $d G / d z=0$ (e.g., Tailleux and McWilliams 2001). As this bottom boundary condition makes analytic expressions for WKB depth variations of $N(z)$ problematic (Tailleux and McWilliams 2001), they will not be pursued. However, the modified boundary condition is straightforwardly implemented in the numerical eigensolution calculation. Surface-intensified vertical

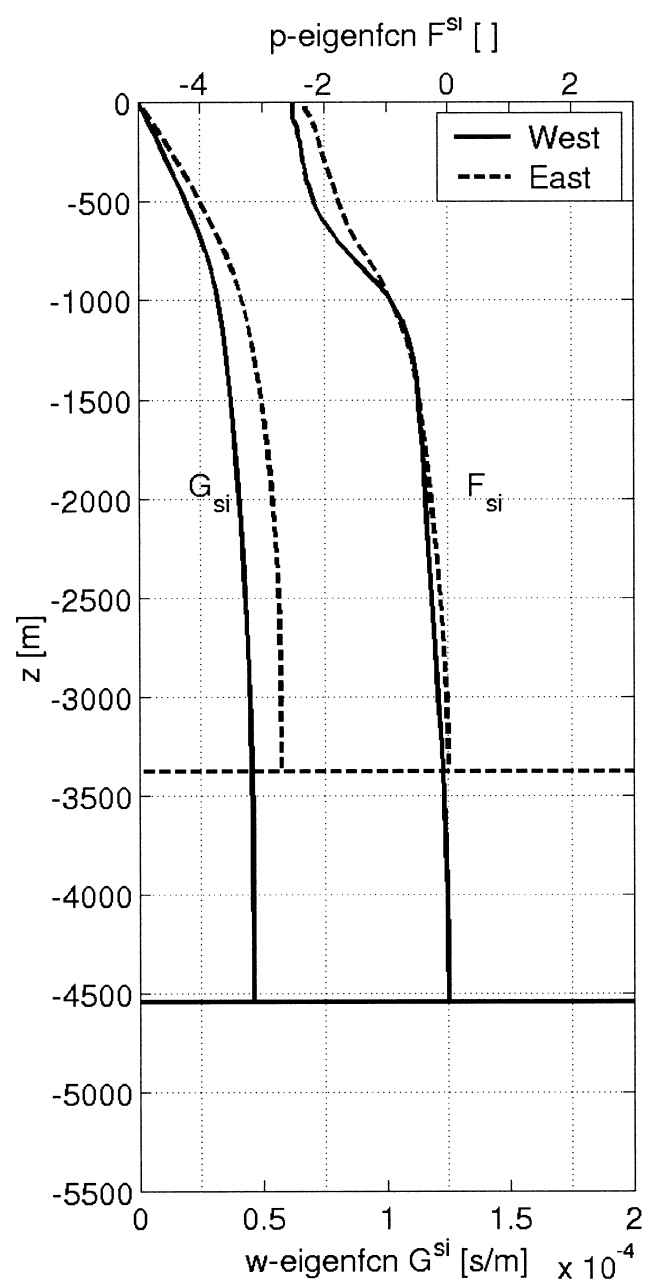

FIG. 4. Vertical eigenfunctions from two North Atlantic locations, as in Fig. 2b, for the surface-intensified mode.

mode structure (Fig. 4) differs fundamentally from that of the standard mode (Fig. 2): $G_{\mathrm{si}}$ takes its maximum at the seafloor and $F_{\mathrm{si}}$ reaches zero at the seafloor with no water-column zero crossing. Comparing Fig. 4 to Fig. $2 \mathrm{~b}$ reveals that near the surface, $\left|d G_{\mathrm{si}} / d z\right|$ is much smaller that $|d G / d z|$, meaning, by (3), that the similar values that $\left|F_{\mathrm{si}}(0)\right|$ and $|F(0)|$ take are only possible because of higher $c_{\mathrm{si}}$ than $c$.

The geographic structure of $\gamma_{\mathrm{si}}$ and its main contributing factors $I_{\mathrm{si}}$ and $c_{s i}^{2}$ (Fig. 5) show certain similarities to those discussed in the previous section for the standard mode. Except at high latitudes, a general increase from northeast to southwest dominates $\gamma_{\mathrm{si}}$ and is largely due to $I_{\text {si }}$, while patterns in $c_{s i}^{2}$ and $H$ appear to compensate each other with less influence on $\gamma_{\mathrm{si}}$.

The ratios $\gamma_{\mathrm{si}} / \gamma, I_{\mathrm{si}} / I$, and $c_{s i}^{2} / c^{2}$ (Fig. 6) make clear specifically how the surface-intensified mode results differ from those of the standard mode. The value of $\gamma_{\mathrm{si}}$ exceeds that of $\gamma$ everywhere except a small southwest region. This is particularly true over shallow areas, and as a result, the pattern in $\gamma_{\mathrm{si}}$ (Fig. 5a) does not show 

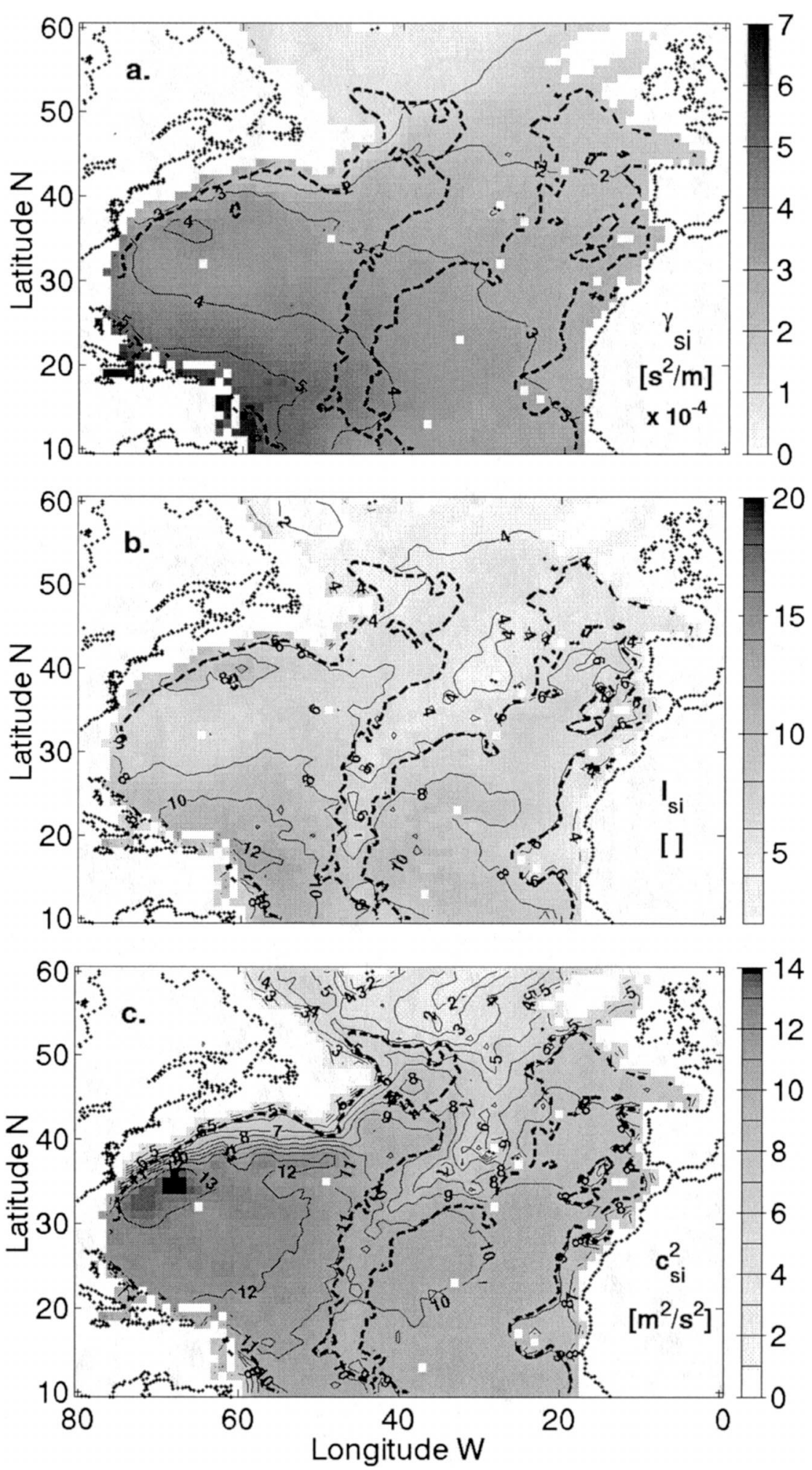

FIG. 5. Surface-intensified mode results: (a) $\gamma_{\mathrm{si}}$ (cf. Fig. 1b), (b) $I_{\mathrm{si}}$ (cf. Fig. 3a), and (c) $c_{\mathrm{si}}^{2}$ (cf. Fig. 3b). 

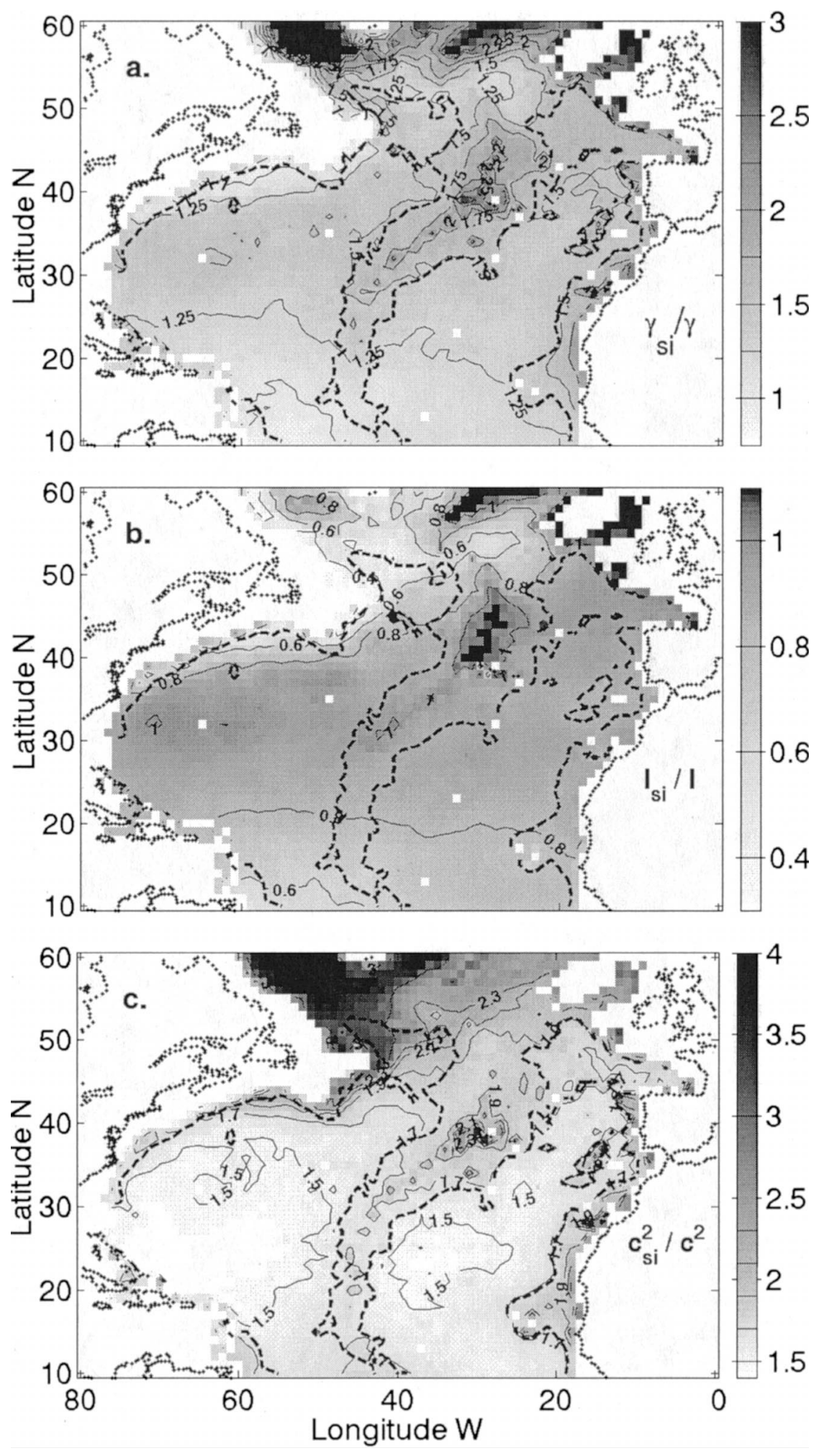

FIG. 6. Ratios of surface-intensified to standard vertical mode parameters: $(a)=(b) \times(c)$; (a) $\gamma_{\mathrm{si}} / \gamma$, (b) $I_{\mathrm{si}} / I$, and (c) $c_{\mathrm{si}}^{2} / c^{2}$. 
relatively low values over shallow topography to the same extent that $\gamma$ does (Fig. 1b). This is consistent with the natural interpretation for the surface-intensified mode that it is less sensitive to the bottom topography. The underlying cause for the increased magnitudes of $\gamma_{\mathrm{si}}$ over $\gamma$ is seen to be increases in $c_{s i}^{2}$ over $c^{2}$, which overcome the fact that $I_{\mathrm{si}}$ is generally less than $I$ (Figs. $6 b, c)$.

To summarize, for the surface-intensified mode the ratio between SSHA variance and wave energy across the North Atlantic shows relatively minor differences with that for the standard mode: slightly higher values over most of the basin and a less severe local reduction of values over the midocean ridge. These attributes are due mainly to a higher internal gravity wave speed and reduced sensitivity to bathymetry, respectively.

\section{Conclusions and discussion}

Effects of geographic variations in vertical eigensolution structure on first baroclinic mode Rossby waves have been considered with respect to the relation between SSHA variance and vertically integrated energy and to the efficiency of Ekman pumping in exciting wave SSHA. To summarize, two parameters are identified as important, the ratio $\gamma(11)$ between squared SSHA and wave energy, and the unitless coefficient $\alpha$ [a multiple of $\gamma ;(14 \mathrm{~b})$ ] of the Ekman pumping term in the forced wave equation for SSHA. An expression (12) for $\gamma_{\mathrm{WKB}}$ applicable to an $N(z)$ profile with depth variations weak in the WKB sense shows that it is proportional to the water depth as well as the surface and vertical-mean buoyancy frequency values. Using climatological hydrographic data (Gouretski and Jahncke 1998) to numerically calculate (Chelton et al. 1998) first-mode eigensolutions demonstrates that $\gamma$ (and hence $\alpha$ ) vary by roughly an order of magnitude across the North Atlantic (Fig. 1b). Largest values occur in the south and west and smallest values at northern latitudes, as controlled by spatial variations in the normalized squared surface value of the pressure eigenfunction and in the internal gravity wave speed. Treatment of the surface-intensified mode (Rhines 1970; Samelson 1992; Tailleux and McWilliams 2001; and others) in place of the standard vertical mode shows similar results except that $\gamma_{\mathrm{si}}$ (Figs. 5 and 6) has slightly higher values and is less strongly reduced over shallow water.

Using $\gamma$ and measured annual-frequency propagating SSHA, the geographic variation in vertically integrated first baroclinic mode wave energy has been inferred in the North Atlantic (Fig. 1c). At $35^{\circ} \mathrm{N}$, the SSHA variance (Fig. 1a) in the western half of the basin is $63 \%$ higher than that in the eastern half, while the westward increase in inferred wave energy for the standard vertical mode (Fig. 1c) is just 26\%; inferred energy increases westward $41 \%$ as much as SSHA variance. (One can make these same east-west comparisons in terms of SSHA and square root of wave energy, as opposed to
SSHA variance and wave energy. At this latitude, the westward increases are then reduced to $28 \%$ and $12 \%$, respectively, so the square root of energy increases $42 \%$ as much as the SSHA. For either choice, the use of a geographically uniform relation between altimetric signal and energy metric invites misinterpretation.) The surface-intensified vertical mode shows smaller eastwest $\gamma_{\mathrm{si}}$ contrasts, but the westward inferred energy increase remains only $57 \%$ as high as for SSHA variance in this example.

These results demonstrate and quantify the importance of geographic variations in stratification and bathymetry to interpretation of SSHA and wind forcing of baroclinic Rossby waves. For example, while increased SSHA variance to the west of midocean ridges (e.g., CS; PC) has helped to motivate theory and modeling (e.g., Pedlosky 2000; Tailleux and McWilliams 2000), westward increases in inferred wave energy are less dramatic. In addition, suitable incorporation of a spatially variable coefficient $\alpha$ could potentially benefit modeling of SSHA patterns (e.g., Vivier et al. 1999; Wang et al. 2001; Birol and Morrow 2001) by use of the long-wave equation forced by measured Ekman pumping.

Acknowledgments. Comments from R. Tailleux and an anonymous reviewer led to many improvements. Discussions with P. Polito and I. da Silveira were important early in the project. We thank P. Hemenway for assisting with altimeter data processing. Supported through the NSCAT program of the National Aeronautics and Space Administration (No. 957627 via the Jet Propulsion Laboratory). Salary for PC provided by the State of Rhode Island and Providence Plantations.

\section{REFERENCES}

Benada, R., 1997: Merged geophysical data record (TOPEX/Poseidon) Generation B User's Handbook Version 2.0. JPL PO.DAAC D11007, Jet Propulsion Laboratory, Pasadena, CA. [Available online at http://podaac.jpl.nasa.gov/woce/woce3_topex/topex/docs/ html/usr_toc.htm.]

Birol, F., and R. Morrow, 2001: Source of the baroclinic waves in the southeast Indian Ocean. J. Geophys. Res., 106 (C5), 91459160 .

Chelton, D. B., and M. G. Schlax, 1996: Global observations of oceanic Rossby waves. Science, 272, 234-238.

— - R. A. de Szoeke, M. G. Schlax, K. E. Naggar, and N. Siwertz, 1998: Geographical variability of the first baroclinic Rossby radius of deformation. J. Phys. Oceanogr., 28, 433-460.

Dewar, W. K., 1998: On "too fast" baroclinic planetary waves in the general circulation. J. Phys. Oceanogr., 28, 1739-1758.

Emery, W. J., W. G. Lee, and L. Magaard, 1984: Geographic and seasonal distributions of Brunt-Väisälä frequency and Rossby radii in the North Pacific and North Atlantic. J. Phys. Oceanogr., 14, 294-317.

Frankignoul, C., P. Muller, and E. Zorita, 1997: A simple model of the decadal response of the ocean to stochastic wind forcing. $J$. Phys. Oceanogr., 27, 1533-1546.

Gill, A., 1982: Dynamics of Atmospheres and Oceans. Academic Press, $662 \mathrm{pp}$.

Gouretski, V. V., and K. Jahncke, 1998: A new climatology of the 
World Ocean. Tech. Rep. 162/98, WOCE Hydrographic Programme Special Analysis Centre (SAC), Hamburg, Germany. [Available online at http://www.dkrz.de/ u241046/SACserver/ SACHome.htm.]

Halliwell, G. R., J. R. Ro, and P. Cornillon, 1991: Westward-propagating SST anomalies and baroclinic eddies in the Sargasso Sea. J. Phys. Oceanogr., 21, 1664-1680.

Killworth, P. D., and J. R. Blundell, 1999: The effect of bottom topography on the speed of long extratropical Rossby waves. $J$. Phys. Oceanogr., 29, 2689-2710.

_ D. Chelton, and R. A. de Szoeke, 1997: The speed of observed and theoretical long extratropical planetary waves. J. Phys. Oceanogr., 27, 1946-1966.

LaCasce, J. H., 2000: Baroclinic Rossby waves in a square basin. $J$. Phys. Oceanogr., 30, 3161-3178.

Levitus, S., R. Burgett, and T. P. Boyer, 1994: Salinity. Vol. 3, World Ocean Atlas 1994, NOAA Atlas NESDIS 3, 99 pp.

Lighthill, J., 1978: Waves in Fluids. Cambridge University Press, 504 pp.

Pedlosky, J., 1987: Geophysical Fluid Dynamics. 2d ed. SpringerVerlag, $710 \mathrm{pp}$

- 2000: The transmission and transformation of baroclinic Rossby waves by topography. J. Phys. Oceanogr., 30, 3077-3101.

Polito, P. S., and P. Cornillon, 1997: Long baroclinic Rossby waves detected by TOPEX/POSEIDON. J. Geophys. Res., 102 (C2), 3215-3235.

— - O. T. Sato, and W. T. Liu, 2000: Characterization and validation of the heat storage variability from TOPEX/Poseidon at four oceanographic sites. J. Geophys. Res., 105 (C7), 16 911-16 921.

Price, J., and L. Magaard, 1980: Rossby wave analysis of the baroclinic potential energy in the upper 500 meters of the North Pacific. J. Mar. Res., 38, 249-264.

Qiu, B., W. Miao, and P. Müller, 1997: Propagation and decay of forced and free baroclinic rossby waves in off-equatorial oceans. J. Phys. Oceanogr., 27, 2405-2417.
Rhines, P. B., 1970: Edge-, bottom-, and Rossby waves in a rotating stratified fluid. Geophys. Astrophys. Fluid Dyn., 1, 273-302.

Rossby, C.-G., 1939: Relation between variations in the intensity of the zonal circulation of the atmosphere and the displacements of the semipermanent centers of action. J. Mar. Res., 2, 38-55.

Samelson, R. M., 1992: Surface-intensified Rossby waves over rough topography. J. Mar. Res., 50, 367-384.

Stammer, D., 1997: Steric and wind-induced changes in TOPEX/ POSEIDON large-scale sea surface topography observations. $J$. Geophys. Res., 102 (C9), 20 987-21 009.

Sturges, W., B. G. Hong, and A. J. Clarke, 1998: Decadal wind forcing of the North Atlantic subtropical gyre. J. Phys. Oceanogr., 28, 659-668.

Tailleux, R., and J. C. McWilliams, 2000: Acceleration, creation, and depletion of wind-driven, baroclinic Rossby waves over an ocean ridge. J. Phys. Oceanogr., 30, 2186-2213.

, and - 2001: The effect of bottom pressure decoupling on the speed of extratropical, baroclinic Rossby waves. J. Phys. Oceanogr., 31, 1461-1476.

Vivier, F., K. A. Kelly, and L. Thompson, 1999: Contributions of wind forcing, waves, and surface heating to sea surface height observations in the Pacific Ocean. J. Geophys. Res., 104 (C9), $20767-20788$.

Wang, L. P., C. J. Koblinsky, and S. Howden, 2001: Annual Rossby wave in the southern Indian Ocean: Why does it "appear" to break down in the middle ocean? J. Phys. Oceanogr., 31, 5474.

White, W., 1977: Annual forcing of baroclinic long waves in the tropical North Pacific. J. Phys. Oceanogr., 7, 50-61.

Wunsch, C., 1997: The vertical partition of oceanic horizontal kinetic energy. J. Phys. Oceanogr., 27, 1770-1794.

— waves in Pacific sea level variations. Deep-Sea Res., 23, 371390.

and D. Stammer, 1997: Atmospheric loading and the oceanic "inverted barometer" effect. Rev. Geophys., 35, 79-107. 